\title{
Agente comunitário de saúde: reflexões sobre o processo de trabalho em saúde em tempos de pandemia de Covid-19
}

\author{
Community health workers: reflections on the health work process \\ in Covid-19 pandemic times
}

Fernanda Beatriz Melo Maciel (https://orcid.org/0000-0002-6421-3940) ${ }^{1}$

Hebert Luan Pereira Campos dos Santos (https://orcid.org/0000-0003-2722-7945) ${ }^{1}$

Raquel Araújo da Silva Carneiro (https://orcid.org/0000-0003-3837-2814) ${ }^{1}$

Eliana Amorim de Souza (https://orcid.org/0000-0002-9653-3164) ${ }^{1}$

Nília Maria de Brito Lima Prado (https://orcid.org/0000-0001-8243-5662) ${ }^{1}$

Carmen Fontes de Souza Teixeira (https://orcid.org/0000-0002-8080-9146) ${ }^{2}$

${ }^{1}$ Instituto Multidisciplinar em Saúde, Universidade Federal da Bahia (UFBA). R. Hormindo Barros 58, Candeias. 45029-094 Vitória da Conquista BA Brasil.fernandamlmcl728@ gmail.com

${ }^{2}$ Instituto de Saúde

Coletiva, UFBA. Salvador

BA Brasil.
Abstract Abstract This study discusses the reorganization of the Community Health Workers (CHWs) work process as a result of the Covid-19 pandemic, considering its importance as a link between the community and the health services in the field of basic care. The literature review comes from the following databases: Virtual Health Library, Scientific Electronic Library Online, and the Brazilian Scientific Publications Portal databases in open access and document review of technical and normative notes from the Municipal Health Secretariats in Brazil. The analysis was based on the premises of Primary Health Care and on the axes of the CHW work, especially cultural competence and community orientation, aiming to discuss the changes introduced in this work regarding the following aspects: 1) health teams support, 2) use of telehealth, and 3) health education. This study concluded that the Covid-19 pandemic demanded reorganization of the work process and assistance flows in the field of basic care. In order for the CHW to continue developing their activities it is necessary to guarantee decent working conditions, training and continuing education, including the concern about the possible discontinuity of other care needed to ensure the population health care in the territory.

Key words Coronavirus infections, Pandemics, Community health workers, Primary health care
Resumo O objetivo deste estudo é discutir a reorganização do processo de trabalho do Agente Comunitário de Saúde (ACS) em decorrência da pandemia da Covid-19, considerando sua importância enquanto um elo de articulação entre a comunidade e os serviços de saúde. Foi realizada uma revisão de literatura nas bases de dados Biblioteca Virtual em Saúde, Scientific Electronic Library Online e Portal Brasileiro de Publicações Científicas em acesso aberto e revisão documental de notas técnicas e normativas das secretarias estaduais de saúde do Brasil. A análise baseouse nas premissas da Atenção Primária à Saúde e nos eixos do trabalho do ACS, especialmente a competência cultural e a orientação comunitária, buscando discutir as mudanças introduzidas neste trabalho no que diz respeito aos seguintes aspectos: apoio às equipes de saúde, utilização da telessaúde e educação em saúde. Concluiu-se que a pandemia de Covid-19 demandou reorganização do processo de trabalho e dos fluxos assistenciais, e para que o ACS continue desenvolvendo suas atividades deve-se garantir condições dignas de trabalho, capacitação e educação permanente, evidenciando-se inclusive, a preocupação quanto à possível descontinuidade de outros cuidados necessários para garantir a atenção à saúde da população no território.

Palavras-chave Infecções por Coronavírus, Pandemia, Agente comunitário de saúde, Atenção Primária à Saúde 


\section{Introdução}

A reforma do modelo assistencial em saúde no Brasil, preconizada pelo Movimento Sanitário ${ }^{1}$, culminou, ao final da década de 1980, na criação do Sistema Único de Saúde (SUS), pautado nos princípios da universalidade, integralidade, equidade, descentralização, regionalização e participação social².

O processo de construção do SUS ${ }^{3}$ tem contemplado, ao longo dos últimos 32 anos, a experimentação de diversas propostas alternativas de organização dos serviços, especialmente no âmbito da atenção básica ${ }^{3,4}$, entre as quais destaca-se a implantação do Programa de Agentes Comunitários de Saúde - PACS, a partir de 1991, e o Programa de Saúde da Família (PSF), criado em 1994 que, posteriormente, passa a ser considerado como Estratégia de Saúde da Família (ESF1997).

Nesse processo, a categoria profissional do Agente Comunitário de Saúde (ACS) se vincula ao PSF, para atuar nas unidades básicas e constituir o elo entre a comunidade e os serviços de saúde ${ }^{5}$. Salienta-se que a ESF é a estratégia prioritária para consolidação e ampliação da atenção básica, a qual, através da Política Nacional de Atenção Básica (PNAB), sob Portaria no 2436/2017, determina como princípios e diretrizes: "o exercício de práticas de cuidado e gestão, democráticas e participativas, trabalho em equipe, dirigido a populações de territórios definidos, compreendendo o sujeito em sua singularidade e inserção sociocultural, em busca de uma atenção integral"'.

O ACS, portanto, é fundamental na Atenção Primária à Saúde (APS) por possuir como atributos do seu trabalho a competência cultural, a orientação comunitária e a construção de vínculo, relacionando-se cotidianamente com as famílias do seu território e transitando entre os saberes técnicos e populares ${ }^{7,8}$.

Diante da pandemia pelo novo coronavírus (SARS-CoV-2), as unidades que operacionalizam a ESF, ao ofertarem um atendimento territorializado e promoverem a longitudinalidade e a coordenação do cuidado em todos os níveis de atenção à saúde, possuem a capacidade de identificar antecipadamente potenciais casos graves ${ }^{9}$. Contudo, para atender a essa demanda, o processo de trabalho em saúde precisou ser significativamente readequado, em consequência das restrições logísticas e espaço-temporais, tais como novas formas de desenvolvimento de atividades pela equipe na APS e o próprio isolamento social da comunidade. Tais mudanças comprometem o trânsito entre os diferentes territórios de abrangência das equipes da ESF, o vínculo presencial com os usuários/comunidade, especialmente, no contexto das visitas domiciliares, assim como exigiu mudanças nos fluxos assistenciais e na interrelação entre os membros da equipe, dificultando, inclusive, a realização de reuniões presenciais para o planejamento mensal de atividades 9 .

Nesse sentido, o presente trabalho busca sistematizar algumas reflexões acerca das estratégias adotadas para a requalificação do trabalho do ACS, com manutenção dos atributos essenciais da APS, em tempos de enfrentamento da pandemia da COVID-19. Este tema se mostra relevante na medida em que a pandemia pelo coronavírus demanda uma reconfiguração dos processos de trabalho em saúde, em especial, o desenvolvido pelo ACS no território, o qual requer, como já mencionado, o vínculo, o contato e o reforço dos tributos derivados da APS, especialmente, a mobilização e a orientação dos diversos grupos populacionais, para a promoção e a proteção da saúde, no contexto da crise sanitária.

\section{Estratégia metodológica}

Estudo teórico-reflexivo construído através da análise crítica, de textos específicos identificados por meio de revisão da literatura científica e documental, incluindo notas, recomendações e orientações referentes à reorganização do processo de trabalho do ACS no contexto de pandemias, especificamente pelo Sars-CoV-2.

Para a seleção dos estudos, realizou-se uma busca no dia 09 de junho de 2020, nas bases bibliográficas Biblioteca Virtual em Saúde (BVS), Scientific Electronic Library Online (SciELO) e Portal Brasileiro de Publicações Científicas em acesso aberto (Oasis) usando como descritores e operadores boleanos os termos: "agente comunitário de saúde AND covid-19", "agente comunitário de saúde AND infecções por coronavírus", "agente comunitário de saúde AND pandemia AND atenção primária à saúde", "agente comunitário de saúde AND infecções por coronavírus OR covid-19 AND atenção primária à saúde" e seus correspondentes em inglês.

Em seguida incluiu-se a revisão documental em plataformas digitais e sites institucionais, tais quais o Ministério da Saúde (MS) e os sites oficiais das Secretarias de Saúde dos 26 estados brasileiros e Distrito Federal sendo incluídos documentos, normativas e notas que tivessem como 
objetivo orientar o trabalho destes profissionais no contexto da pandemia. Adicionalmente, com o mesmo objetivo, foram examinadas as páginas na Internet de portais específicos para o coronavírus da Organização Mundial da Saúde (OMS), Organização Panamericana de Saúde (OPAS), Fundação Oswaldo Cruz (Fiocruz) e Associação Brasileira de Saúde Coletiva (Abrasco).

O corpus de análise constituiu-se de 9 artigos e 14 documentos técnicos, incluindo notas, recomendações e orientações referentes à reorganização do processo de trabalho do ACS no contexto da pandemia de COVID-19. Salienta-se que a análise dos sites das 26 Secretarias Estaduais de Saúde e DF revelaram que apenas 10 estados (37\%) publicaram normas e/ou orientações de trabalho aos ACS no contexto da pandemia, sendo estes os estados da Bahia, Espírito Santo, Mato Grosso, Minas Gerais, Goiás, Rio Grande do Norte, Rio Grande do Sul, Santa Catarina, São Paulo e Paraíba. Verificou-se também que o MS emitiu dois documentos, um de recomendação e outro de orientação, voltados aos ACS em tempos de pandemia por coronavírus. Do mesmo modo, a Fiocruz e a Abrasco também realizaram publicações nesse sentido. Outras instituições como a OMS e a OPAS não emitiram arquivos específicos aos agentes de saúde, abrangendo recomendações gerais aos profissionais de saúde que atuam na linha de frente.

O referencial teórico para subsidiar tal discussão baseia-se no conceito de processo de trabalho em saúde ${ }^{10-12}$. Para tanto, as categorias centrais de análise pautaram-se em aspectos relacionados à produção do cuidado pelos ACS antes e durante a pandemia norteado pelas premissas da APS: acessibilidade, integralidade, longitudinalidade e coordenação do cuidado ${ }^{13}$.

\section{Resultados e Discussão}

\section{O trabalho do agente comunitário de saúde: premissas e tessitura na APS}

A inserção do ACS está prevista em legislações ${ }^{6,14-16}$ e a sua integração na ESF, como membro da equipe multiprofissional, redirecionou seu processo de trabalho, que passou a ter como foco as famílias sob responsabilidade das equipes da APS, as quais, por intermédio da ESF, privilegiam a "promoção, proteção e recuperação de saúde, fomentada de forma integral e continuada" 17 , envolvendo a "longitudinalidade, integração e coordenação do cuidado" 17 .
A atenção primária contempla o acolhimento, a garantia da acessibilidade e a utilização dos serviços de saúde pelos usuários, a cada nova necessidade ou a cada novo episódio de um problema de saúde, considerando os aspectos geográficos, organizacionais, socioculturais e econômicos ${ }^{6}$. Essa vinculação prevê a continuidade do cuidado e a longitudinalidade da atenção à saúde, independentemente da presença de problemas específicos relacionados à saúde ou do tipo de problema ${ }^{17}$. Através dessa vinculação busca-se construir caminhos para uma abordagem integral do indivíduo e das famílias, incluindo a articulação entre os diversos serviços e ações de saúde para disponibilizar um conjunto de serviços e informações que respondam a suas necessidades de saúde de forma integrada, por meio de diferentes pontos da rede de atenção à saúde ${ }^{13}$.

É nesse cenário que as atribuições do ACS incluem a competência cultural e a orientação comunitária, tendo a educação em saúde como o eixo principal do trabalho nos diversos territórios. A competência cultural permite o "reconhecimento das características culturais dos grupos sociais e de suas diferentes necessidades e concepções do processo saúde-doença" ${ }^{18}$, sendo decisiva frente a melhor eficiência e efetividade do trabalho do ACS na APS. A orientação comunitária, por sua vez, é entendida como a capacidade de unir competências epidemiológicas e clínicas a fim de fundamentar programas para melhor reconhecer as demandas emergentes em saúde da população adscrita ${ }^{19}$.

A partir de 2017, a Portaria no 2.436 de 21 de setembro ${ }^{6}$ definiu as seguintes atribuiçõos do ACS: estimular a participação da comunidade nas políticas públicas; orientar as famílias quanto ao uso adequado dos serviços de saúde; identificar, por meio de visitas domiciliares periódicas e monitoramento das famílias, situações de risco; aferição da pressão arterial, medição de glicemia capilar, aferição de temperatura axilar durante a visita domiciliar, encaminhar casos e situações de risco identificados aos outros membros das equipes de saúde; auxiliar no planejamento e implementação das ações de saúde tanto localmente, ao encaminhar informações do território de abrangência para as ESF, quanto nacionalmente, alimentando dados dos sistemas de informação do Ministério da Saúde (MS) ${ }^{6}$.

É válido reconhecer, oportunamente, que apesar do trabalho do ACS ser considerado do campo da saúde envolve ações intersetoriais, a exemplo do contato com a rede socioassistencial, através da articulação com o Centro de Referên- 
cia de Assistência Social (CRAS) e do Centro de Referência Especializado de Assistência Social (CREAS), ao buscar melhores estratégias para a prevenção e a promoção da saúde das famílias adscritas às equipes da $\operatorname{ESF}^{20}$.

Considerando a Portaria no $2.436^{6}$ e compreendendo a competência cultural e a orientação comunitária como eixos basilares do trabalho do ACS, percebe-se que as atribuições preconizadas enfatizam uma atuação distanciada de uma APS efetiva ${ }^{21}$, concebida como porta de entrada e eixo organizador do cuidado, na medida em que prevê a execução de atividades burocráticas pelo ACS, em detrimento da prevenção de riscos e promoção da saúde das famílias e da população que vive e trabalha no território de abrangência das unidades de $\mathrm{SF}$ às quais está vinculado.

\section{Reorganização do trabalho do ACS em contextos de emergências em saúde pública}

A atuação profissional no campo da saúde é orientada por modelos logísticos de prestação de serviços nos diferentes âmbitos e realidades, determinando assim as atividades, as técnicas e os instrumentos que podem ser empregados em cada profissão ${ }^{12}$. A APS possui como eixos centrais para reordenação do cuidado em saúde a organização e a gestão dos processos de trabalho ${ }^{12}$, desse modo, torna-se relevante compreender os componentes desse processo e como eles se (re) organizam em cenários de emergência em saúde pública.

O processo de trabalho em saúde é compreendido como resultado da soma de processos pelos quais indivíduos atuam utilizando meios de produção, sobre algum objeto para, modificando-o, obterem determinado resultado/produto ${ }^{10-12}$. No âmbito da APS, reconhece-se que os ACS integram o conjunto dos agentes; os objetos são as condições ou necessidades sociossanitárias em saúde dos indivíduos e famílias; os meios de produção ou instrumentos de trabalho são os conhecimentos, as habilidades, as ferramentas e/ou equipamentos em geral; e as finalidades ou objetivos são projeções de resultados que visam a satisfazer necessidades e expectativas, conforme sua organização social, em dado momento histórico ${ }^{10-12}$.

Ao considerar o processo de trabalho em saúde a partir desse conceito, os eventos de emergência em saúde pública requerem, para além de novas formas de comportamento social, a adequação e o desenvolvimento de estratégias e metodologias de trabalho, especialmente, na atuação dos ACS. Carmo et al. ${ }^{22}$ apontam que, em contextos como esse, há "necessidade de readequação e aprimoramento de conceitos, estruturas, processos e práticas de vigilância em saúde, de forma articulada com a rede de atenção à saúde". Nesse sentido, a COVID-19 representa um dos maiores desafios sanitários dos últimos anos, influenciando diretamente na dinâmica e na relação entre os elementos que compõem esse processo de trabalho, alterando também a prestação do cuidado em saúde no território.

Nesse contexto, o objeto de trabalho dos ACS ultrapassa as necessidades de saúde que já existiam no território, passando a incluir as novas demandas que surgem a partir da situação de emergência e requerem o acionamento de outros, como: aquisição de saberes, aperfeiçoamento de práticas e utilização de novas ferramentas, como as tecnologias de informação e comunicação e as mídias sociais.

Os atributos do trabalho do ACS - competência cultural e orientação comunitária - entretanto, não podem ser excluídos do cotidiano dos serviços. Em períodos de pandemia ou surtos, essas características, inclusive, auxiliam na maior permeabilidade e aceitação das medidas estabelecidas e preconizadas pelas autoridades de saúde do país a nível territorial. Isso se deve não apenas à capilaridade que o ACS possui no território, mas, sobretudo, por esse trabalhador ser um dos principais responsáveis no reconhecimento das demandas e peculiaridades do território de sua responsabilidade. Ademais, a função de vigilância em saúde tem sido apontada como a chave para minimizar danos e reduzir as mortes evitáveis em situações de emergências em saúde pública ${ }^{9}$.

Ao revisar as evidências ${ }^{23-27}$ sobre o trabalho do ACS em contextos pandêmicos, aponta-se que estes trabalhadores representam parte significativa da força de trabalho na linha de frente e podem ser importantes no controle e na prevenção de pandemias como a COVID-19, sendo observado que, em países com programas bem estabelecidos, as funções e as tarefas do ACS mudam substancialmente durante pandemias, haja vista a necessidade de redefinição dos procedimentos normais de condução das atividades rotineiras. Recomenda-se que além de treinamento, supervisão e apoio, estes profissionais recebam orientação adaptada e atualizada continuamente ${ }^{23-27}$.

No cenário internacional, são consideradas atividades comuns dos ACS durante pandemias: consciência, engajamento e sensibilização da comunidade sobre aspectos das doenças, adoção de medidas de segurança e combate ao estigma, além de rastreamento de contatos ${ }^{23}$. 
A partir disso e da exploração do material selecionado, as reflexões acerca da reorganização do trabalho no contexto dessa epidemia pelo SARS-CoV-2 foram conduzidas com base em categorias teórico empíricas, tais quais: 1) Apoio às equipes de saúde, 2) Utilização de Telessaúde e 3) Educação em saúde. Optamos por aprofundar o debate a partir desses três tópicos por considerar que são os que mais se sobressaem. Adicionalmente, construímos um diagrama (Figura 1), a ser explicado ao longo das seções que se seguem, que exemplifica a reestruturação do processo de trabalho do ACS no contexto da pandemia da COVID-19, sem o desvincular das premissas que estruturam a APS.

\section{Apoio às equipes de saúde}

A reorientação do processo de trabalho do ACS em apoio às equipes de saúde deve ocorrer de modo a cumprir as premissas da APS, como apresentado no diagrama, mas no cenário da pandemia a acessibilidade e a longitudinalidade/ continuidade do cuidado em saúde tornam-se primordiais para a garantia da assistência ${ }^{28}$. Considerando as atribuições já definidas na Portaria $\mathrm{n}^{\circ} 2.436$, identificamos que a estruturação das atividades do ACS em apoio às equipes de saúde inclui visita domiciliar e coleta de dados; diagnóstico situacional de pacientes vulneráveis e assistência a pacientes com doenças crônicas e vigi-

Processo de Trabalho do ACS em contexto de emergência em saúde pública
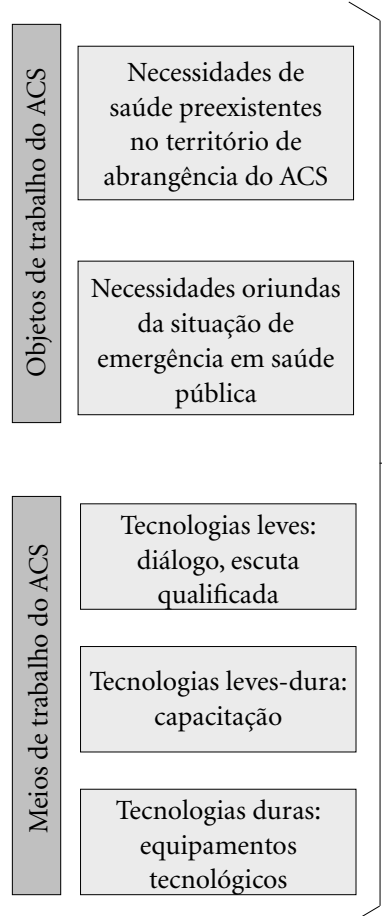

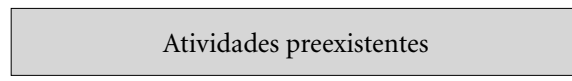

Informar a população sobre a busca de cuidado em caso de urgências

Informar a população sobre a busca de cuidado em caso de urgências

Organizar o fluxo de acolhimento dos usuários, presencialmente e por teleatendimento

Auxiliar a equipe na identificação de casos suspeitos e grupos vulneráveis/ de risco

Realizar busca ativa de novos casos suspeitos

Orientar a população sobre a doença e medidas de prevenção

Eixos basilares: Orientação comunitária e competência cultural

Integração entre a equipe de saúde e população adscrita

Busca ativa e notificação de doenças

Orientar a família quanto à utilização dos serviços de saúde disponíveis

Promover a mobilização e participação comunitária

Vigilância à saúde, através das visitas domiciliares

Garantir a integralidade da atenção realizando ações de pomoção da saúde e prevenção de agravos

Identificar parceiros e recursos na comunidade que possam potencializar ações intersetoriais com a equipe

Ações educativas nos domicílios e na comunidade

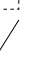

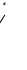


lância ativa e passiva dos casos leves confirmados para COVID-19,29-38.

No que tange à visita domiciliar, preconiza$\mathrm{se}^{9,29-38}$ a abstenção em adentrar nos domicílios e a priorização do ambiente peridomiciliar para a execução das atividades previstas. Oportunamente, é orientado utilizar este momento para indagar a quantidade de cômodos do domicílio, o número de pessoas residentes, considerando a faixa etária e a presença de comorbidades, assim como as condições de acesso ao saneamento básico, de modo a realizar, preliminarmente, uma análise de risco quanto ao coronavírus ${ }^{29}$. Haines et al. ${ }^{25}$ e Ballard et al. ${ }^{26}$ apontam que as visitas domiciliares são oportunidades chave para diagnóstico situacional das vulnerabilidades, pois permite identificar usuários/famílias em extrema pobreza, insegurança alimentar, assim como grupos vulneráveis. Chatterjee ${ }^{27}$ chama atenção para o caráter intersetorial que é demandando, uma vez que, ao identificar os mais vulneráveis, o ACS pode acionar outras instâncias do sistema de saúde e dos serviços de proteção e assistência social, para garantir que esses grupos recebam apoio alimentar, monetário, social e médico necessário, seja provido por órgãos governamentais ou por outras fontes.

Do ponto de vista territorial, com base nas informações domiciliares coletadas e catalogadas, é designado aos ACS mapear usuários de maior risco para COVID-19, acionando redes de apoio social. Do mesmo modo, registrar e analisar a distribuição de casos de COVID-19 por local de moradia, sexo, faixa etária, presença de comorbidades e vulnerabilidade social ${ }^{39}$. Nesse sentido, por estar em contato direto com o território, orienta-se aos ACS a precisão de mapear possibilidades de locais ou estratégias de isolamento para usuários de maior risco para a COVID- $19^{39}$.

Goldfield et al. ${ }^{40}$ consideram que as atividades desenvolvidas devem incluir o monitoramento das mudanças na situação epidemiológica e nas estratégias de saúde pública, visando sua aceitação social, controle de novos casos, rastreamento de contatos e monitoramento de saúde, prevenção e controle rigoroso de infecções em instalações de saúde, uso de outras intervenções ativas de controle, atividades contínuas de vigilância ativa e compartilhamento de dados com os demais membros da equipe de saúde e sistemas de informação do país, atribuições essas que já são preconizadas pela PNAB.

Haines et al. ${ }^{25}$ mencionam que a atuação dos ACS junto aos territórios e comunidade contribui para a construção do vínculo e confiança no sistema de saúde, de modo a permitir que no futuro estes se envolvam em testes comunitários para COVID-19, inclusive na ampliação de testes diagnósticos. A longo prazo, podem contribuir para o gerenciamento de condições através do monitoramento da saúde física e mental e da revisão da disponibilidade e uso de medicamentos ${ }^{25}$.

No âmbito da Unidade de Saúde, o ACS fica incumbido de organizar o fluxo de acolhimento de modo a evitar aglomeração de grupos com mais de 10 pessoas, acolhendo os usuários, preferencialmente em ambientes arejados, além de auxiliar nas atividades de vigilância ativa e passiva, fluxos sobre tratamentos e calendários de vacinação $0^{38}$. Do mesmo modo, as recomendações brasileiras, estabelecem que o ACS deve auxiliar no atendimento através do Fast-track COVID-19, ferramenta de fluxo rápido de triagem e atendimento de casos de síndrome gripal com método derivado de protocolos de triagem em situações de emergências ${ }^{38}$.

Estas recomendações ${ }^{9,38}$ incluem, além das ações de promoção, prevenção e controle de agravos, atribuições relacionadas à identificação e monitorização de casos suspeitos e confirmados através da avaliação de temperatura, pressão arterial e detecção precoce de sintomas da COVID-19. Contudo, é essencial o fornecimento de equipamentos de proteção individual (EPI) e outros equipamentos (termômetro, oxímetros de pulso portáteis), assim como o devido treinamento para os ACS estarem qualificados e seguirem de forma eficaz e resolutiva, os protocolos indicados na situação da pandemia ${ }^{9,38}$.

$O$ desafio que tem sido posto não se restringe ao trabalho do ACS, mas abrange o processo de trabalho na APS, para garantir que os cuidados contínuos aos usuários não sejam interrompidos e que os pacientes do território não sejam desassistidos, e paralelamente definir fluxos específicos para usuários com suspeita ou confirmação da COVID-19 durante a pandemia.

Por outro lado, uma característica fulcral que envolve o trabalho do ACS, calcado nas premissas da acessibilidade e longitudinalidade, é o alcance de populações marginalizadas e vulneráveis para garantir que tenham acesso aos serviços, sobretudo, a testes e quaisquer resultados acionáveis de forma oportuna com mensagens culturalmente adequadas e necessárias para o enfrentamento da pandemia ${ }^{9,29-38}$.

\section{Utilização da Telesaúde}

Diante da necessidade de adoção de medidas de distanciamento social e restrição à realização 
das visitas domiciliares, em caráter de excepcionalidade reconheceu-se a possibilidade e a eticidade da utilização da telessaúde como prioridade, desde que possível, para a atuação dos $\mathrm{ACS}^{29-38}$.

A telessaúde é compreendida, nesse sentido, como um sistema de prestação de serviços de saúde à distância, intermediado por tecnologias da informação e comunicação (TICs) ${ }^{41}$. Silva $^{42}$ descreve-a como uma forma de conceber os processos de saúde, quebrando a barreira da distância a partir do uso das TICs, abrangendo subáreas, como tele-educação sanitária ou em saúde (utilização das TICs para desenvolver atividades educacionais à distância, sejam elas palestras, cursos, conferências, web-aulas); redes de investigação e tele-epidemiologia (aplicação das TICs para fins de monitoramento e investigação epidemiológica com a utilização de sistemas vinculados a internet); redes de administração e gestão em saúde, assim como a telemedicina, a qual envolve a teleorientação, o telemonitoramento (orientação e supervisão para monitoramento ou vigência à distância de parâmetros de saúde e/ou doença), a teleconsultoria e o telediagnóstico ${ }^{41}$.

Cabe destacar que, apesar de mais fortemente estimulada no contexto atual, essa ferramenta vem sendo regulamentada pelo MS: em 2006 foi criada a Comissão Permanente de Telessaúde e o Comitê Executivo de Telessaúde; em 2007, o Programa Nacional de Telessaúde Brasil Redes e o Projeto Nacional de Telessaúde, o qual posteriormente foi substituído, através da Portaria no 2.546, em 2011, pelo Telessaúde Brasil Redes, que objetiva fortalecer e melhorar a qualidade do atendimento da atenção básica no SUS ${ }^{43}$.

Dentre as recomendações e orientações dos documentos técnicos avaliados para a adequação do trabalho do ACS na situação da pandemia sugeriu-se como possibilidade a realização de "visitas online”, em 'substituição' às visitas domiciliares, intermediada por canais de comunicação (whatsapp, email, telefone e outros) da ESF e ou de APS para monitorar, comunicar a população sobre o cancelamento de consultas e orientar sobre a rotina de trabalho da UBS na situação vigente ${ }^{38}$.

A Secretaria de Saúde do Rio Grande do Sul ${ }^{36}$, por exemplo, propôs que o trabalho fosse realizado, de forma intercalada, entre visitas domiciliares e monitoramento telefônico de usuários com sintomas da COVID-19; mais especificamente, a cada 24hs, a pessoas com mais de 60 anos e portadores de condições clínicas de risco e a cada $48 \mathrm{hs}$ aos demais, até completar 14 dias do início dos sintomas e, caso necessário, orientar atendi- mento presencial. Haines et al. ${ }^{25}$, consoante ao proposto pela Secretaria do Rio Grande do Sul, apresenta a possibilidade da revisão regular dos grupos considerados vulneráveis, pessoalmente ou virtualmente, dependendo da necessidade.

A Abrasco ${ }^{44}$ ressalta que para cumprir a efetividade das ações desenvolvidas pelos ACS na dimensão virtual faz-se necessário o desenvolvimento de capacidades no uso da internet e aplicativo. Cabe destacar que as $\mathrm{PNABs}^{6,16}$ já garantiam liberdade aos municípios para desenvolver ou adquirir softwares para coletar ou gerenciar dados das famílias, da mesma forma a disponibilidade e a manutenção dos tablets ou smartphones para ESF e ACS. Mais recentemente, o MS, buscando melhorar a qualidade da informação em saúde, tem conduzido o processo de implementação da Estratégia e-SUS AB através da informatização dos serviços de saúde e incremento de tecnologias para viabilizar o processo de trabalho na $\mathrm{AB}^{45}$.

Contudo, Abreu et al. ${ }^{46}$ destacam aspectos que dificultam a implantação desses sistemas, principalmente devido à ausência do suporte para esclarecimento de dúvidas, bem como a de manuais para os ACS, o que acaba originando percepções negativas na operacionalização dessas ferramentas. Mendonça et al. ${ }^{47}$ ressaltam que, diante desse cenário, é preciso investigar a cobertura da utilização das TICs pela comunidade de atuação de cada ACS, pois, ainda hoje, o acesso a essas ferramentas se dá de modo heterogêneo entre os diversos grupos sociais, o que impede a inclusão de grande parte da população, especialmente os mais pobres e menos letrados, na sociedade digital, e restringe, desse modo, a possibilidade do ACS de acompanhá-los com base nessa metodologia.

Se reconhece que a tele-educação, a tele-epidemiologia e o telemonitoramento, apesar de já estarem sendo incorporadas ao trabalho do ACS, têm sido mais exigidos em seu cotidiano, no momento atual ${ }^{46,47}$. A incorporação desses novos instrumentos de trabalho requer investimentos estatais para a capacitação, a aquisição e a manutenção dos aparelhos e aplicativos utilizados, assim como o reconhecimento das desigualdades sociais e de acessibilidade no que diz respeito à utilização dessas tecnologias para o processo de trabalho do $\mathrm{ACS}^{47}$. Assim, deve-se atentar para que a inserção desses novos meios de trabalho aumente a eficiência e a efetividade do trabalho do ACS e favoreça a longitudinalidade e a acessibilidade aos cuidados prestados pela APS. 


\section{Educação em saúde}

As medidas de educação em saúde, até então, ocorreram de forma hierarquizada e sobrecarregada de uma visão estritamente tecnicista, resultando em campanhas e materiais elaborados na perspectiva puramente biomédica ${ }^{48}$. Entretanto, ao reconhecer que os processos de saúde-doença-cuidado se expressam de formas diferentes, de acordo com o grupo social atingido e são determinados por dimensões macro e microestruturais, essas ferramentas não são consumidas e compreendidas por todos com a mesma avidez ${ }^{48}$.

A atuação do ACS já possuía como essencialidade o trabalho educativo visando prevenção de doenças e promoção da saúde às famílias e às comunidades. No atual contexto, a imprescindibilidade dessa ação foi reforçada em todos os documentos analisados ${ }^{9,29-38}$. Apontam, sobretudo, a necessidade de se fortalecer competência do ACS em fomentar, na comunidade, a compreensão sobre a situação epidemiológica, formas de transmissão do vírus, sinais e sintomas da COVID-19, bem como de fornecer orientações e informações sobre o funcionamento da UBS, medidas de proteção comunitárias, domiciliares e pessoais, como distanciamento social, uso de máscaras de tecido, higiene pessoal, lavagem de mãos, etiqueta respiratória, com atenção especial para dirimir fake news s,29-38. $^{9}$.

O ACS, como educador em saúde, possui a árdua tarefa de contribuir com reflexões junto às iniciativas populares, sociais e do campo da saúde no enfrentamento às situações que a pandemia traz, assim como de coletar, conhecer, sistematizar e traduzir as angústias e as dúvidas da população neste cenário ${ }^{49}$. Para isso, a utilização das tecnologias, mídias digitais (como grupos de WhatsApp), meios de comunicação locais, como bicicleta com caixa de som, rádios comunitárias, carros de som, jornais comunitários, folhetos informativos e cartazes são possibilidades para conduzir a ação nesse período ${ }^{27,50}$.

A pandemia da COVID-19, nesse aspecto, tem revelado a necessidade de estratégias educacionais que alcancem as crenças pessoais e a visão de mundo amplamente influenciadas por fatores históricos, culturais e sociais, uma vez que estas determinam os comportamentos e as escolhas individuais, as quais podem, neste momento, ser um desafio para os profissionais que estão diretamente envolvidos no enfrentamento da SARSCoV $-2^{27}$.

Dada a competência comunitária ser um dos diferenciais do trabalho do ACS, o diálogo com a população sobre suas demandas e a importância de fomentar a participação social e o engajamento comunitário torna-se fundamental ${ }^{48}$. Devese destacar que o ACS tem assumindo papel de liderança, embora não de forma sistemática, na promoção de estratégias de comunicação aprimoradas $^{25,40}$.

No ambiente da unidade de saúde, o ACS fica encarregado de realizar atividades educativas enquanto os pacientes aguardam atendimento, orientando sobre a utilização das ferramentas disponíveis, como o aplicativo Coronavírus-SUS do MS, bem como de reforçar as orientações aos pacientes que ficarão em isolamento e aos seus cuidadores $^{28}$. Apesar do atual cenário epidemiológico, ressalta-se que as epidemias de arboviroses não deixaram de existir, logo é fundamental que os ACS aproveitem as oportunidades supracitadas para orientar sobre medidas de prevenção da dengue, zika e chikungunya, bem como sintomas e busca por atendimento na unidade de saúde ${ }^{29-38}$.

\section{Considerações necessárias}

É nítido que a pandemia da COVID-19 demandou reestruturação dos sistemas de saúde e reorganização do processo de trabalho e dos fluxos assistenciais, no entanto, essa reorganização da APS não pode significar descontinuidade de outros cuidados prevalentes no território, principalmente em cenários fragilizados e com vulnerabilidades tão distintas presentes no tecido social brasileiro e que se expressam na heterogeneidade da situação epidemiológica da população nos diversos territórios. Almeja-se que a experiência advinda do enfrentamento deste evento crítico, cujos múltiplos efeitos nas condições de vida e saúde da população brasileira ainda estão em progressão catastrófica, possa estimular o reconhecimento e a mobilização da sociedade em defesa do SUS, e da promoção do seu fortalecimento e maior preparo, em especial, no contexto da APS, para que em situações como essa, o trabalho e os fluxos de cuidado das equipes possam ser reajustados rapidamente sem comprometer as ações que já vinham sendo desenvolvidas para intervir sobre os determinantes do processo saúde-doença e sobre os riscos que as famílias e os indivíduos adscritos estão sujeitos.

$\mathrm{O}$ incremento de novos meios de trabalho, como o uso das TICs, deve ser ampliado para além dos momentos de emergência em saúde pública, visando, sobretudo, potencializar e oti- 
mizar as atividades desenvolvidas. Por fim, para o desenvolvimento do modelo apresentado na figura é preciso garantir condições dignas de trabalho, capacitação e educação permanente do ACS, porquanto não se pode esquecer que o trabalho desenvolvido pelo ACS é primordial para a execução das premissas que orientam a APS brasileira. Não se pode deixar que o trabalho que possui como eixos basilares a orientação comunitária e a competência cultural seja substituído por outras racionalidades, como um trabalho burocrático e inefetivo na prestação do cuidado em saúde.

Considerando os aspectos supracitados, apresentamos a seguir algumas recomendações direcionadas ao processo de reorganização do trabalho do ACS em situações de emergência sanitária, com base na reflexão sobre a experiência do enfrentamento da COVID-19:

Desenvolvimento de uma perspectiva de educação em saúde que não atribua aos indivíduos e às famílias a "culpa" pela exposição aos riscos de adoecimento e morte, e que não seja meramente prescritiva de mudanças de hábitos e atitudes, senão que reconheça o papel desempenhado na determinação do processo saúde-doença pelas condições de vida, trabalho e lazer;

Desenvolvimento e construção de estratégias de comunicação culturalmente acessíveis através de orientações que sejam alcançáveis a todas as parcelas da população, com treinamento e supervisão contínuos dos ACS para qualificá-los no uso dessas novas estratégias de comunicação e educação em saúde;

Garantia de equipamentos de proteção individual e outras condições de trabalho e salário dignos e oferta de apoio psicológico ao ACS e sua família;

Tais recomendações pautam-se na premissa da qualificação profissional para viabilizar as situações de necessária reorganização do processo de trabalho do ACS, sem prejuízos às perspectivas legais, de modo a garantir a oferta de ações e serviços à população adscrita, a busca de universalidade do acesso e a da equidade em saúde, em defesa da dignidade do trabalho e da vida do ACS, dos trabalhadores de saúde em geral e da população.

\section{Colaboradores}

FBM Maciel, HLPC Santos, RA Silva, EA Souza, NMBL Padro e CFS Teixeira contribuíram substancialmente na concepção e no planejamento do estudo; na obtenção, na análise e interpretação dos dados e na redação e revisão crítica e aprovaram a versão final a ser publicada.

\section{Agradecimentos}

Traduzido por integrantes do Grupo de tradução voluntária de materiais informativos relacionados ao projeto COVID-19, oferecido pelo NUPEL/UFBA e orientado pelos professores M. Daniel Vasconcelos, Dra. Feibriss Henrique Meneghelli Cassilhas, Dra. Lucielen Porfírio e Dra. Monique Pfau. Tradutores: Simone Maria Evangelista Salles e Fernanda da Silva Góis Costa. 
1. Paim JS. S. A reforma sanitária como objeto de reflexão teórico-conceitual. In: Paim JS. Reforma sanitária brasileira: contribuição para a compreensão e crítica. Salvador, Rio de Janeiro: EDUFBA, Fiocruz; 2008. p. 153-174.

2. Fraga OS. Agente Comunitário de Saúde: Elo entre a comunidade e a equipe ESF. Especialização em Atenção Básica em Saúde da Família. Governador Valadares: Universidade Federal de Minas Gerais; 2011.

3. Teixeira CF, Vilasboas ALQ. Desafios da formação técnica e ética dos profissionais das equipes de Saúde da Família. In: Trad L, organizador. Família contemporânea e Saúde: significados, práticas e políticas públicas. Rio de Janeiro: Fiocruz; 2010. p. 133-156.

4. Paim JS. Modelos de atenção à Saúde no Brasil. In: Giovanella L, Escorel S, Lobato LVC, Noronha JC, Carvalho AI, organizadores. Políticas e Sistema de Saúde no Brasil. Rio de Janeiro: Fiocruz, CEBES; 2008. p. 547-573.

5. Barros DF, Barbieri AR, Ivo ML, Silva MG. O contexto da formação dos agentes comunitários de saúde no Brasil. Texto contexto - enferm 2010; 19(1):78-84.

6. Brasil. Ministério da Saúde (MS). Portaria no 2.436, de 21 de setembro de 2017. Aprova a Política Nacional de Atenção Básica, estabelecendo a revisão de diretrizes para a organização da Atenção Básica, no âmbito do Sistema Único de Saúde. Diário Oficial da União 2017; 21 set.

7. Maciazeki-Gomes RC, Souza CD, Baggio L, Wachs F. O trabalho do agente comunitário de saúde na perspectiva da educação popular em saúde: possibilidades e desafios. Cien Saude Colet 2016; 21(5):1637-1646.

8. Alonso CMC, Béguin PD, Duarte FJCM. Trabalho dos agentes comunitários de saúde na Estratégia Saúde da Família: metassíntese. Rev Saude Publica 2018; 52:14.

9. Brasil. Ministério da Saúde (MS). Recomendações para Adequação das Ações dos Agentes Comunitários de Saúde Frente à Atual Situação Epidemiológica Referente ao Covid-19. Brasília: MS; 2020.

10. Ramos MN. Conceitos Básicos sobre Trabalho. In: Fonseca AF, organizador. O processo histórico do trabatho em saúde. Rio de Janeiro: Fiocruz; 2007. p. 27-56.

11. Lima JC. Bases histórico-conceituais para a compreensão do trabalho em saúde. In Fonseca AF, organizador. O processo histórico do trabalho em saúde. Rio de Janeiro: Fiocruz; 2007. p. 57-96.

12. Faria HP, Werneck MA, Santos MA, Teixeira PF. Processo de Trabalho em Saúde. os Santos. Universidade Federal de Minas Gerais/ Nescon. 2009; 2ed.

13. Starfield B. Atenção primária: equilíbrio entre necessidades de saúde, serviços e tecnologia. Brasília: Ministério da Saúde; 2002

14. Brasil. Ministério da Saúde (MS). Portaria nº. 648, de 28 de março de 2006. Política nacional de atenção básica. Diário Oficial da União 2006; 26 mar.

15. Brasil. Ministério da Saúde (MS). Lei no 10.507 de 10 de julho de 2002. Cria a profissão de Agente Comunitário de Saúde e dá outras providências. Diário Oficial da União 2002; 10 jul.

16. Brasil. Ministério da Saúde (MS). Política Nacional de Atenção Básica. Brasília: MS; 2012.
17. Oliveira MA, Pereira IC. Atributos essenciais da Atenção Primária e a Estratégia Saúde da Família. Rev Bras Enferm 2013; 66(n. esp):158-164.

18. Gouveia AH, Silva R, Pessoa BH. Competência Cultural: uma Resposta Necessária para Superar as Barreiras de Acesso à Saúde para Populações Minorizadas. Revista Brasileira de Educação Médica 2019; 43(1 Supl. 1):82-90.

19. De Paula WK, Samico IC, Caminha MF, Filho MB, Figueirôa JN. Orientação comunitária e enfoque familiar: avaliação de usuários e profissionais da estratégia saúde da família. Cad Saúde Colet 2017; 25(2):242248.

20. Medeiros J. Os desafios da intersetorialidade no âmbito do SUAS. Viçosa: GESUAS; 2017.

21. Morosini MV, Fonseca AF, Lima LD. Política Nacional de Atenção Básica 2017: retrocessos e riscos para o Sistema Único de Saúde. Saúde Debate 2018; 42(116):1124.

22. Carmo EH, Penna G, Oliveira WK. Emergências de saúde pública: conceito, caracterização, preparação e resposta. Estudos Avançados 2008; 22(64):19-32.

23. Bhaumik S, Moola S, Tyagi J, Nambiar D, Kakoti M. Community health workers for pandemic response: a rapid evidence synthesis. BMJ Global Health 2020; 5:e002769.

24. Gondim G, Nogueira M. Orientações para ACS, ACE e AVS no território-domicílio ou território-moradia em casos de quarentena e suspeitos por Covid-19. Rio de Janeiro: SES/RJ; 2020.

25. Haines A, Barros EF, Berlin A, Heymann DL, Harris MJ. National UK programme of community health workers for COVID-19 response. Lancet 2020; 395(10231):1173-1175.

26. Ballard M, Bancroft E, Nesbit J, Johnson A, Holeman I, Foth J, Rogers D, Yang J, Nardella J, Olsen H, Raghavan M, Panjabi R, Alban R, Malaba S, Christiansen M, Rapp S, Schechter J, Aylward P, Rogers A, Sebisaho J, Ako C, Choudhury N, Westgate C, Mbeya J, Schwarz $\mathrm{R}$, Bonds $\mathrm{MH}$, Adamjee R, Bishop J, Yembrick A, Flood D, McLaughlin M, Palazuelos D. Prioritising the role of community health workers in the COVID-19 response. BMJ Global Health 2020; 5:e02550.

27. Chatterjee PK. Community preparedness for COVID-19 and frontline health workers in Chhattisgarh. Indian J Public Health 2020; 64(Supl.):S102-S104.

28. Santa Catarina. Secretaria de Estado da Saúde de Santa Catarina (SES/SC). Nota Técnica no 003/2020 DAPS/SPS/SES/SC. Florianópolis: SES/SC; 2020.

29. Goiás. Secretaria de Estado da Saúde de Goiás (SES/ GO). Nota Técnica no: 14/2020 - SAIS- 03083. Goiânia: SES/GO; 2020

30. São Paulo. Secretaria de Estado da Saúde de São Paulo (SES-SP). Orientações para a organização das ações no manejo do novo coronavírus (COVID-19) na Atenção Primária à Saúde. São Paulo: SES-SP; 2020.

31. Rio Grande do Norte. Secretaria de Saúde Pública do Rio Grande do Norte (SESAP). Nota Técnica $n^{\circ}$ 14/2020/SESAP. Natal: SESAP; 2020. 
32. Espírito Santo. Secretaria de Estado da Saúde do Espírito Santo (SESA). Nota Técnica COVID-19 n 19/2020 SESA/SSAS/GROSS/NEAPRI. Vitória: SESA; 2020.

33. Bahia. Secretaria da Saúde da Bahia (SESAB). Nota Técnica - novo Coronavírus, No 01 de 16/03/2020. Assunto: orientações para organização da Atenção Básica (ab), no enfrentamento do novo coronavírus. Salvador: SESAB; 2020.

34. Gondim G, Nogueira M. Orientações para ACS, ACE e AVS no território-domicílio ou território-moradia em casos de quarentena e suspeitos por Covid-19. Rio de Janeiro: SES; 2020.

35. Minas Gerais. Escola de Saúde Pública do Estado de Minas Gerais (ESP-MG). ESP-MG lança novo curso em EaD: ACS no Enfrentamento da Covid-19. Belo Horizonte: ESP-MG; 2020.

36. Rio Grande do Sul. Departamento de Ações em Saúde (DAS). Coordenação Estadual da Atenção Básica. Recomendações para a organização interna das equipes de Atenção Básica do RS frente à pandemia do COVID-19. Porto Alegre: DAS; 2020.

37. Paraíba. Secretaria da Saúde da Paraíba. Manejo Clínico APS. 2020. [acessado 2020 Jul 13]. Disponível em: https://paraiba.pb.gov.br/diretas/saude/coronavirus/ profissionais-de-saude/orientacoes-do-ministerioda-saude

38. Brasil. Ministério da Saúde (MS). Orientações para a organização das ações no manejo do novo coronavírus (COVID-19) na Atenção Primária à Saúde. Brasília: MS; 2020.

39. Fiocruz. ACS destacam-se por seu papel de educadores em meio à crise do coronavírus. 2020. [acessado 2020 Jul 15]. Disponível em: http://www.epsjv.fiocruz.br/ podcast/acs-destacam-se-por-seu-papel-de-educadores-em-meio-a-crise-do-coronavirus

40. Goldfield NI, Crittenden R, Fox D, McDonough J, Nichols L, Lee Rosenthal E. COVID-19 Crisis Creates Opportunities for Community-Centered Population Health: Community Health Workers at the Center. J Ambul Care Manage 2020; 43(3):184-190.

41. Pereira CCA, Machado CJ. Telessaúde no Brasil - conceitos e aplicações. Cien Saude Colet 2015; 20(10):3283-3284.

42. Silva AB. Telessaúde no Brasil - conceitos e aplicações. Rio de Janeiro: Editora DOC; 2014.

43. Morsch JA. Telessaúde Brasil: o que é, como funciona, legislação e benefícios. 2020. [acessado 2020 Jul 15]. Disponível em: https://telemedicinamorsch.com.br/ blog/telessaude-brasil

44. Associação Brasileira de Saúde Coletiva (Abrasco). Fortalecer a Estratégia Saúde da Família no enfrentamento da Covid-19 - Posicionamento da Rede APS, da Abrasco. Rio de Janeiro: Abrasco; 2020.

45. Brasil. Ministério da Saúde (MS). e-SUS Atenção Básica : Manual do Aplicativo ACS - Agente Comunitário de Saúde - Versão 2.0. Brasília: MS; 2015.
46. Abreu F, Zanin N, Bissaco MA, Silva AP, Boschi SR Scardovelli TA, Marni SC. Percepções dos agentes comunitários de saúde sobre as tecnologias de informação e comunicação na Atenção Primária à Saúde: uma pesquisa exploratório. Revista Humanidades e Inovação 2020; 8(5):32-45.

47. Mendonça AV, Sousa MF, Linhares RN, Cabral JC, Paixão PB. Inclusão digital dos agentes comunitários de saúde no Brasil: novas formas de aprender em rede. Tempus - Actas de Saúde Coletiva 2009; 3(1):28-37.

48. Palácio MA,Takenam I. Em tempos de pandemia pela COVID-19: o desafio para a educação em saúde. Vigil. sanit. debate 2020; 8(2):10-15

49. Associação Nacional de Pós-graduação e Pesquisa (ANAPED). Educação popular em tempos de pandemia: todas as certezas são provisórias. Rio de Janeiro: ANPED; 2020.

50. Bornstein VJ, Goldschmidt I. O papel d@s agentes de saúde na mobilização comunitária em tempos de Covid-19. Rio de Janeiro: EPSJV; 2020.
Artigo apresentado em 28/07/2020

Aprovado em 28/07/2020

Versão final apresentada em 30/07/2020 
